\title{
$\equiv$ ACRONYMS $\equiv$
}

\author{
BNDD Bureau of Narcotics and Dangerous Drugs \\ CI Counterintelligence (CIA) \\ CIA Central Intelligence Agency \\ CTC Counter-Terrorism Center (CIA) \\ DCI Director of Central Intelligence \\ DO Directorate of Operations (CIA) \\ DOJ Department of Justice \\ FAS Federation of American Scientists \\ FBI Federal Bureau of Investigation \\ FISA Foreign Intelligence Surveillance Act \\ FISC Foreign Intelligence Surveillance Court \\ FOIA Freedom of Information Act \\ HVD High Value Detainee \\ IG Inspector General (CIA) \\ IOB Intelligence Oversight Board \\ IRA Irish Republican Army \\ IRS Internal Revenue Service \\ KGB Soviet Intelligence Service \\ NSA National Security Agency \\ NSC National Security Council \\ NYPD New York Police Department
}


$=$ THE FAMILY JEWELS $=$

OGC Office of the General Counsel (CIA)

OLC Office of Legal Counsel (DOJ)

PDB President's Daily Brief

PFIAB President's Foreign Intelligence Advisory Board

PR Public Relations

PRB Publications Review Board (CIA)

TSP Terrorist Surveillance Program 
THE FAMILY JEWELS 
